\title{
Effect of LED emission cross-section in indoor visible light communication systems
}

\author{
Huy Quang Nguyen ${ }^{1}$, Joon-Ho Choi ${ }^{1}$, Tae-Gyu Kang ${ }^{2}$, Sang-Kyu Lim², Dae Ho Kim², Moonsoo Kang ${ }^{3}$ and \\ Chung Ghiu Lee ${ }^{1 *}$
}

\begin{abstract}
Visible light communication (VLC) is an optical wireless communication technology that uses visible light emitting diodes (LEDs) as a communication source. Since the LEDs are also used for illumination, the cross-section emission effects of the LEDs need to be analyzed as they apply to indoor VLC channels. In order to evaluate the illumination and communication performance according to the emission cross-section pattern a simple LED model with a quasi-elliptic emission cross-section is proposed and compared to a circular LED model.

The spatial distributions of the received optical power and root mean square delay spread are analyzed through calculation and comparison. The LEDs with a quasi-elliptic emission cross-section provide less fluctuation in the illumination and optical power distribution at the receiving plane. However, the RMS delay spread increases and subsequently the maximum data rate decreases for the quasi-elliptic emission cross-section LEDs. The single transmitter VLC system is found to support at least 17 and $24 \mathrm{Mb} / \mathrm{s}$ for circular and quasi-elliptic emission cross-section LEDs for the entire receiving plane, respectively. The four-transmitter VLC system is found to support at least at 30 and $33 \mathrm{Mb} / \mathrm{s}$ for circular and quasi-elliptic emission cross-section LEDs for the entire receiving plane, respectively.
\end{abstract}

Keywords: Visible light communication, Optical communication, Optical wireless, Light-emitting diodes; illumination

\section{Introduction}

Due to the fast-growing demand for increasing bandwidth and mobility, many new wireless communication technologies have been developed, such as Bluetooth [1], IEEE 802 [2,3], IrDA [4], and ultra-wideband [5]. Among them, optical wireless technologies provide a high level of security and a low level of RF interference without the need for government regulation to impact frequency usage. The optical wireless communication systems including visible light communication (VLC) are reviewed in detail [6]. Out of all the optical wireless communication techniques, VLC method utilizes the visible wavelength range from 380 to $780 \mathrm{~nm}$. The visible lights are emitted from illumination light emitting diodes (LEDs), which are expected to replace present lighting devices using conventional lamps, i.e. incandescent bulbs and

\footnotetext{
* Correspondence: clee@chosun.ac.kr

'Department of Electronic Engineering, Chosun University, Gwangju 501-759, Korea

Full list of author information is available at the end of the article
}

fluorescent lamps, in the near future. The VLC scheme maintains the advantages of optical wireless communications, except that the data rate (DR) is limited to the modulation bandwidth of the illumination LEDs. In addition, it is safe for human eyes and provides low power consumption, long lifetime, and no frequency regulation. Therefore, the VLC scheme shall become a reasonable option for indoor optical wireless communication infra-structures, for example, in offices, hospitals, and other large-scale buildings where artificial lighting is needed. The recently demonstrated VLC systems include a $513 \mathrm{Mbit} / \mathrm{s}$ VLC link using DMT modulation [7], an experimental video transmission using VLC link [8], an Ethernet-VLC interface implementation [9], a hybrid radio-over-fiber and VLC system [10], an EthernetOCDMA system for multi-user VLC [11], a highspeed optical wireless demonstrator [12].

From a communication viewpoint, wireless communication research using illumination LEDs has had some remarkable success, including the calculation of the 
impulse response [13], [14], optical power distribution simulation [15-19], the communication feasibility through experimentation [20,21], and the enhancement of the DR $[22,23]$. A way to enhance modulation bandwidth of the phosphor-based LED has been tried by optical filtering [23]. The phosphor-based LED is usually used for illumination and shows a limited modulation bandwidth due to longer phosphor absorption and relaxation process than the tri-color LED. A blue LED with yellow phosphor shows the modulation bandwidth of a few MHz. Using a blue optical filter, the modulation bandwidth was improved by an order of magnitude from the LED without blue filtering [23]. The overall illumination from different arrangements of the LEDs in arrays has been analyzed [24,25]. The parameters which affect the illumination uniformity (IU) were also analyzed in [24]. The most of previous simulation studies on VLC systems are focused on the development of the simulation program based on circular emission crosssections. However, non-circular LED emission patterns, for example elliptical cross-section emissions, are also worthy of being analyzed in order to assess their effects on the communication and illumination performance.

In this article, we propose a simulation model for use in the analysis of the effects of LED emission crosssections on VLC systems. For the sake of comparison, each LED is assumed to be a point source, i.e. one LED, in order to provide its circular emission cross-section, because the LED dimensions are small compared to the distance from the LEDs to the receiver; they are in a far-field condition. In addition, we analyzed the effects of the emission cross-section of LEDs needed to achieve a reasonable DR with a proposed simple LED model with a non-circular emission cross-section. These results are compared to LED arrays with a circular emission cross-section. We focus on the emission cross-section of LEDs, one of LED physical parameters. Then, we try to find out its effect on the illumination/communication performances.

This rest of the article is organized as follows: "System model" section deals with the optical wireless communication system model. "Quasi-elliptic emission cross-section LED model" section describes the non-circular quasielliptic emission cross-section LED model. "Performance parameters" section presents the performance parameters. The simulation results are described in "Simulation results" section. A discussion regarding multiple wavelengths in VLC systems is presented in "Discussions" section. Finally, conclusion is given at the last section.

\section{System model}

A typical $5 \mathrm{~m} \times 5 \mathrm{~m} \times 3 \mathrm{~m}$ office room is assumed in the model. The VLC link is assumed to be a line-of-sight diffuse link [17]. It is assumed that the LED lamps are installed at a height of $2.7 \mathrm{~m}$ from the floor and the receiver is placed at the height of $0.85 \mathrm{~m}$. Therefore, the distance from the LED lamps to the receiving plane is $1.85 \mathrm{~m}$. We simulate two cases: one-transmitter and four-transmitter. Each lamp is assumed to be composed of one LED. In the one-transmitter case, the transmitter is placed at the center $(2.5,2.5)$. In the four-transmitter case, the positions of transmitters are assumed to be $(1.25,1.25),(1.25,3.75),(3.75,1.25)$, and $(3.75,3.75)$.

We make several assumptions for the simulation. Sun light and other ambient lighting are assumed to be negligible with an appropriate optical filtering and indoor environment. It is also assumed that there are no shadows to disrupt the communication in this system. MATLAB is used to program and simulate the system model. The center luminous intensity for each LED is set at $410 \mathrm{~cd}$. In the case of the LED group with a circular emission cross-section, the semi-angle at half power is assumed to be $30^{\circ}$, since several commercial LEDs have a value of around $30^{\circ}$ [26]. The physical area of the photodiode detector is assumed to be $1 \mathrm{~cm}^{2}$, taken from a commercial photodiode (FDS 1010, ThorLabs) with a bandwidth of $8 \mathrm{MHz}$ and an assumed receiver field of view of $50^{\circ}$. The VLC system does not focus on maintaining a high DR, due to the current limitations in LED modulation bandwidth. Therefore, the use of low-speed components is plausible.

\section{Quasi-elliptic emission cross-section LED model}

Previous simulation studies regarding illuminance distribution have been done on Lambertian and circular light emission LED patterns [15-19]. The contour plots for the illuminance distribution for these LED patterns are comprised of concentric circles. Recently, it has been reported that different LED varieties have different angular intensity distributions; they are phenomenologically modeled through the consideration their various physical factors, such as the LED chip morphology, how the chip is arrayed, the phosphor surface, the reflected light, and the encapsulating lens shape [26]. Therefore, from a practical point of view, some of illumination LEDs are manufactured having Lambertian emission characteristics.

However, due to the reflector and diffuser in the LED lamp design, the final emission pattern from an LED lamp may no longer be Lambertian and become circular due to the combined effects of the LED emission, reflector and diffuser [27]. We can find some examples of LEDs with non-circular emission cross-sections in [26], by adding application-specific optics from the manufacturers, even though the LED chip itself shows a circular emission cross-section.

In order to evaluate the effects of the LED emission cross-section on the illumination and communication 
performance, we present an LED model possessing a noncircular quasi-elliptic emission cross-section and compare it to a conventional Lambertian emission cross-section, whose light emission is a function of the cosine of the viewing angle. Therefore, under the conditions that the receiving plane is not tilted and is in a direction perpendicular to the LEDs, the shape of the emission crosssection is circular at the receiving plane.

The emission pattern can be expressed as the sum of a maximum of two or three Gaussian or cosine-power functions $[13,24]$. By adopting this approach, we can use the horizontal illuminance equation of an LED with a non-circular emission cross-section by adding each illuminance component from each LED [13,24].

We present a simple model of an LED with a quasielliptic cross-section based on the combination of two LEDs with circular emission cross-sections. The target is a surface perpendicular to the $z$ direction, from the center of the two-LED array, as shown in [24]. The equation that determines the distance between two LEDs for a maximally flat irradiance at a surface perpendicular to the $z$ direction from the center of the two-LED array is $[24,28]$ :

$$
d_{0}=\sqrt{\frac{4}{m+3}} z
$$

where $m$ is the Lambertian order, related to the semiangle at half power, $\theta_{1 / 2}$. By comparing the simulation environment $\left(z=1.85 \mathrm{~m}, d_{0}=1.0 \mathrm{~m}, \theta_{1 / 2}=30^{\circ}\right)$ with the conditions given in Equation (1), it is easy to find an equivalent LED model with a non-circular quasi-elliptic emission cross-section. Figure 1 shows the LED separation variation for a maximally flat illumination at $z=1.85 \mathrm{~m}$ according to Equation (1) achieved by varying $\theta_{1 / 2}$.

Figure 2 compares the horizontal illuminance distributions for one LED with a circular emission cross-section and two equivalent LED models with non-circular emission cross-sections using two LEDs. Figure 2(a) shows the contour plot of the horizontal illuminance distribution for the LED with a circular emission cross-section with $\theta_{1 / 2}=30^{\circ}$ at $z=1.85 \mathrm{~m}$. In order to obtain an equivalent LED using two LEDs with $\theta_{1 / 2}=30^{\circ}$, we increased the LED separation (Figure 2(b) for $d=0.3 \mathrm{~m}$ ) and chose $d=1 \mathrm{~m}$ and its quasielliptic emission cross-section, as shown in Figure 2(c). The center of the two LEDs is considered to be the position of the new equivalent LED with the quasi-elliptic emission cross-section. The non-circular quasi-elliptic LED model makes the simulation simple by placing the LEDs in appropriate positions for a non-circular emission cross-section, compared to LED models needing complex mathematical equations [18].

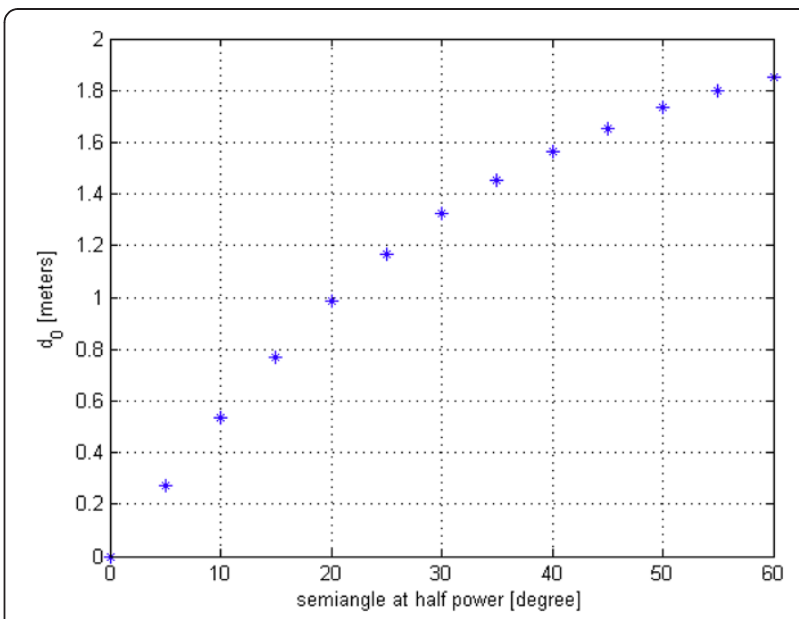

Figure 1 The LED separation variation for maximally flat illumination.

\section{Performance parameters}

\section{Illumination distribution uniformity}

The degree of uniformity needed to measure of the amount of variation in the power on the receiving surface has previously been defined as the ratio of the maximum to minimum received power levels in an indoor optical wireless communication system [15]. This provides the difference between the maximum and minimum optical power values from all of the calculation points. However, since this only calculates two values (maximum and minimum), it does not provide the information regarding how uniform the received optical power (or the illumination at the receiving surface) compares to the average optical power. Therefore, an illumination uniformity (IU) method is proposed as a factor used to determine the horizontal illuminance variations, considering the average illuminance at the receiving surface. IU is therefore defined as the ratio of the difference between the maximum and minimum values of the horizontal illuminance to its average value over the receiving plane, and is given by:

$$
I U=\frac{\left\{E_{i, j}\right\}_{\max }-\left\{E_{i, j}\right\}_{\min }}{\mu_{E}}
$$

where $\left\{E_{i, j}\right\}_{\max }$ denotes the maximum value of the horizontal illuminance at different points on the receiving surface and $\left\{E_{i, j}\right\}_{\min }$ is the minimum value of the horizontal illuminance at different points on the receiving surface. $N$ is the total number of elements in the receiving surface. $\mu_{E}$ denotes the mean of the illuminance and is given by:

$$
\mu_{E}=\frac{1}{N} \sum_{i, j} E_{i, j}
$$

The total number of elements in the receiving surface $N$ is given as $N=N_{x} \times N_{y} . N_{x}$ denotes the 


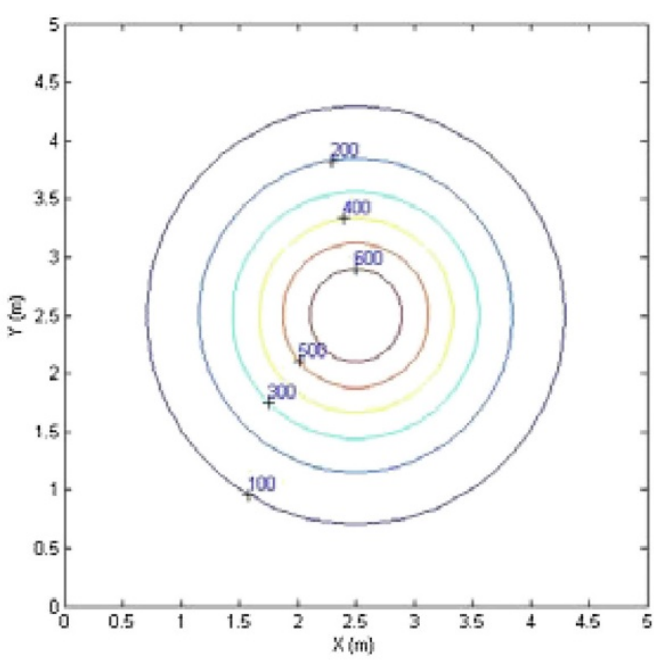

(a)

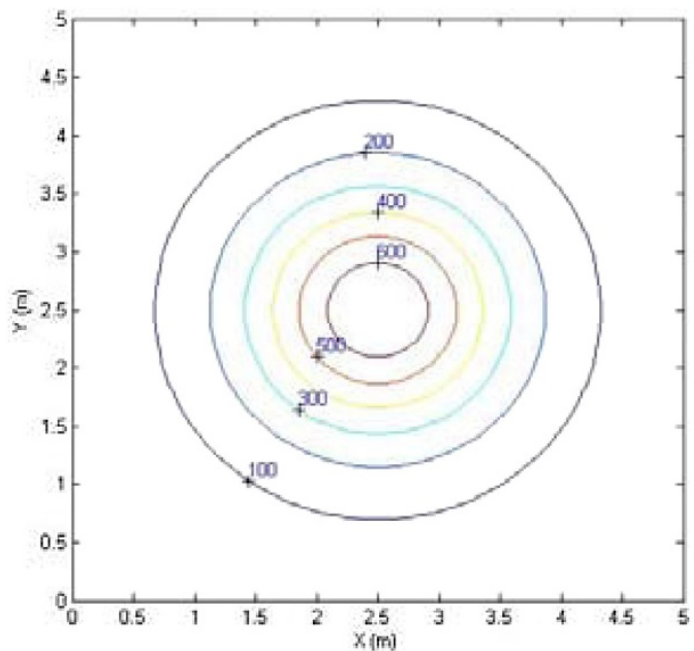

(b)

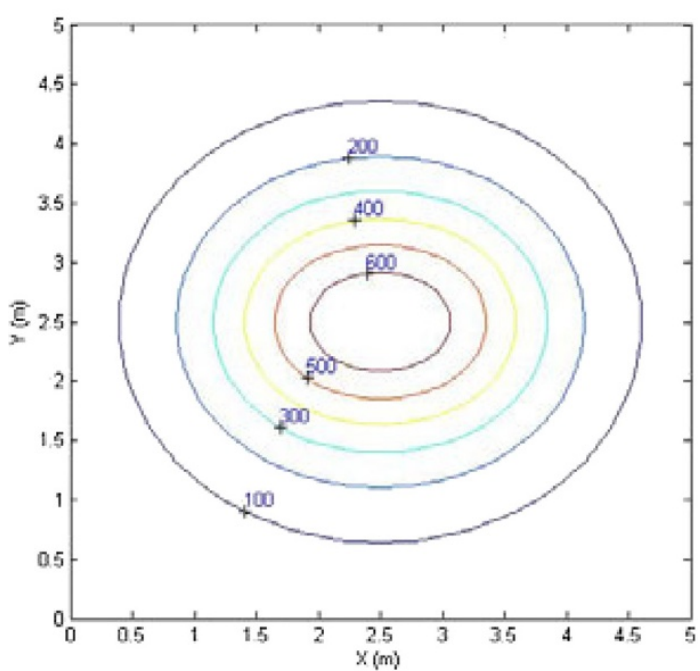

(c)
Figure 2 The horizontal illuminance contour plots: (a) one LED with a circular emission cross-section [Max $683 \mathrm{Ix}$ ], and quasielliptic emission cross-sections with two LEDs separated by a distance: (b) $d=0.3 \mathrm{~m}$ and (c) $d=1.0 \mathrm{~m}$ [Max. $686 \mathrm{~lx}$ ].

number of calculation points along the x-axis; $N_{y}$ denotes the number of calculation points along the y-axis. In the simulations, $N_{x}$ and $N_{y}$ were set to 25 . Figure 3 shows the variation of the IU in a $d B$ scale for the different number of transmitters. The IU depends on the shape of emission cross-section and the semi-angle at half power. When the semi-angle at half power is small, the difference due to the shape of emission cross-section is large. With large semi-angles at half power the effect of the number of transmitters is weak. However, the IU for one transmitter is smaller than that found for four transmitters in the case of large semi-angle at half power.

\section{Root mean square delay spread (RMSDS)}

The channel DC gain on the direct path, the optical gain for the optical concentrator, the channel DC gain, and the distributions of the horizontal illuminance and received optical power are calculated based on the analyses from [13] and [17]. Considering both the direct path and the first-order reflection path, the received optical power at a point can be calculated [17] using:

$$
P_{r}=\sum^{L E D s}\left\{P_{t} \cdot H_{d}(0)+\sum^{\text {reflections }} P_{t} \cdot d H_{\text {ref }}(0)\right\}
$$

where $P_{t}$ is the optical power transmitted from an LED, $H_{d}(0)$ denotes the channel DC gain on the directed paths, and $d H_{r e f}(0)$ is the channel DC gain on the reflection paths $[13,17]$. The transfer function of the channel is given by $H(f)=\int_{-\infty}^{\infty} h(t) e^{-j 2 \pi f t} d t$, where $h(t)$ is the channel impulse response. The average received optical power is given by $P_{r}=H(0)$ $P_{t}$, where $H(0)$ denotes the channel DC gain at $f=0$.

By assuming $M$ direct paths from transmitters to a specific receiver and $N$ reflection paths to the same receiver, the total power of the received optical signals, $P_{T}$, is thereby calculated as:

$$
P_{T}=\sum_{i}^{M} P_{d, i}+\sum_{j}^{N} P_{r, j}
$$

where $P_{d, i}$ is the received optical power of the direct light at the $i$-th point and $P_{r, j}$ is the received optical power of the reflected light at the $j$-th point. $M$ 


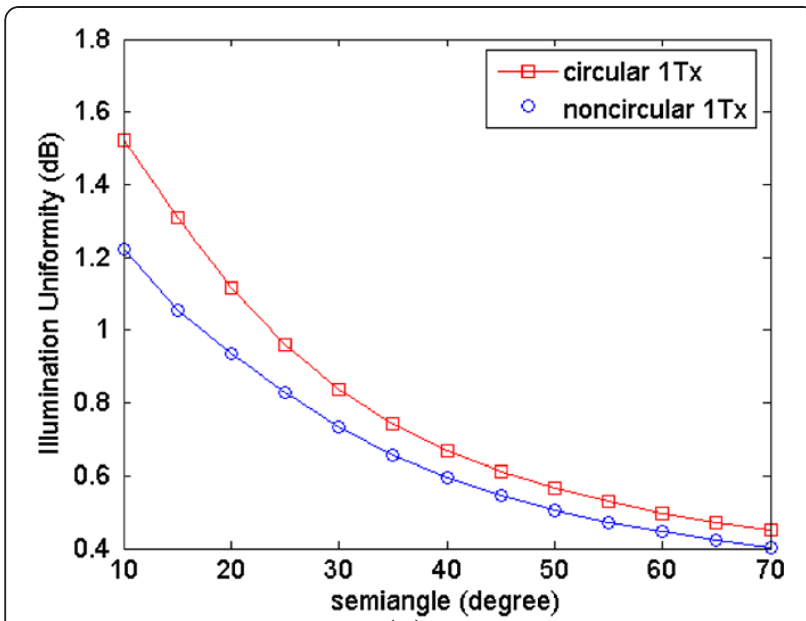

(a)

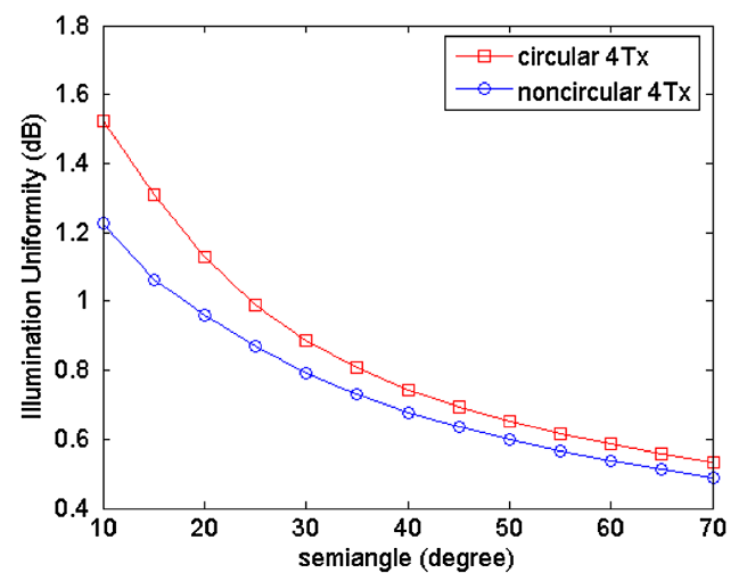

(b)

Figure 3 The LED transmitter uniformity: (a) one-LED and (b) four-LED.

denotes the number of direct light components and $N$ denotes the number of reflected light components.

One of the more important performance parameters in a communication system is the data transmission rate (DTR) or simply the data rate (DR). Usually the maximum DR mainly depends on a combination of the modulation bandwidths of the components used and the channel impulse response. Due to many reflection components caused by walls, the maximum DR for wireless systems is limited. Due to different signal arrival times, the maximum DR at different receiver positions may not be the same. In order to develop some general design guidelines for wireless systems, the RMSDS is used to grossly quantify the multipath channel effects [29]. Similarly, because optical signals experience different path lengths, the optical signal components arrive at a receiver position with different time delays, which cause intersymbol interference (ISI). The RMSDS value provides an estimate for a kind of normalized delay time due to multiple reflections. Therefore, the RMSDS is a critical performance criterion for the upper bound of the DR.

The mean excess delay is defined as [29]:

$$
\bar{\tau}=\left(\sum_{i}^{M} P_{d, i} t_{d, i}+\sum_{j}^{N} P_{r, j} t_{r, j}\right) / P_{T} .
$$

The RMSDS, $\tau_{R M S}$, is given by:

$$
\tau_{R M S}=\sqrt{\overline{\tau^{2}-(\bar{\tau})^{2}}},
$$

where

$$
\overline{\tau^{2}}=\left(\sum_{i}^{M} P_{d, i} t_{d, i}{ }^{2}+\sum_{j}^{N} P_{r, j} t_{r, j}{ }^{2}\right) / P_{T} .
$$

It is noted that the RMSDS depends on the relative levels of the optical power components within $P_{T}$, which is the total optical power determined by Equation. (5).

Figure 4 shows the flowchart used to calculate the RMSDS in the simulation program. The simulation parameters are initialized and the simulation program starts to calculate the direct components and then the reflected components. The calculated data are temporarily stored and post-processed to calculate the RMSDS.

\section{Simulation results}

\section{Effects of emission cross-section on illumination and received optical power}

The distributions of the horizontal illuminance at the distance of $1.85 \mathrm{~m}$ from the LED transmitters and their contour plots used to evaluate the effect of the LED emission patterns in the transmitters are shown in Figure 5. We only take into account the first-order reflections on the walls since the base optical power is significantly greater compared to those found for the higher-order reflection components [13,17]. Figure 5(a) shows the illumination distribution for four transmitters creating a non-circular quasi-elliptic emission cross-section. Each transmitter satisfies the illumination standard set forth by the International Organization for Standardization (ISO). The recommendation states that an illuminance of 300 to $1500 \mathrm{~lx}$ is required for office work [17]. Figure 5(b) shows their contour plots. It can clearly be seen that the transmitters with the quasi-elliptic emission cross-section give less fluctuation in the horizontal illuminance, and therefore, the quasi-elliptic emission cross-section transmitters are superior to those with circular emission cross-sections from the viewpoint of illumination in spite of the asymmetry between the vertical and the horizontal mid lines and the low illumination level in the vertical and horizontal mid lines. 


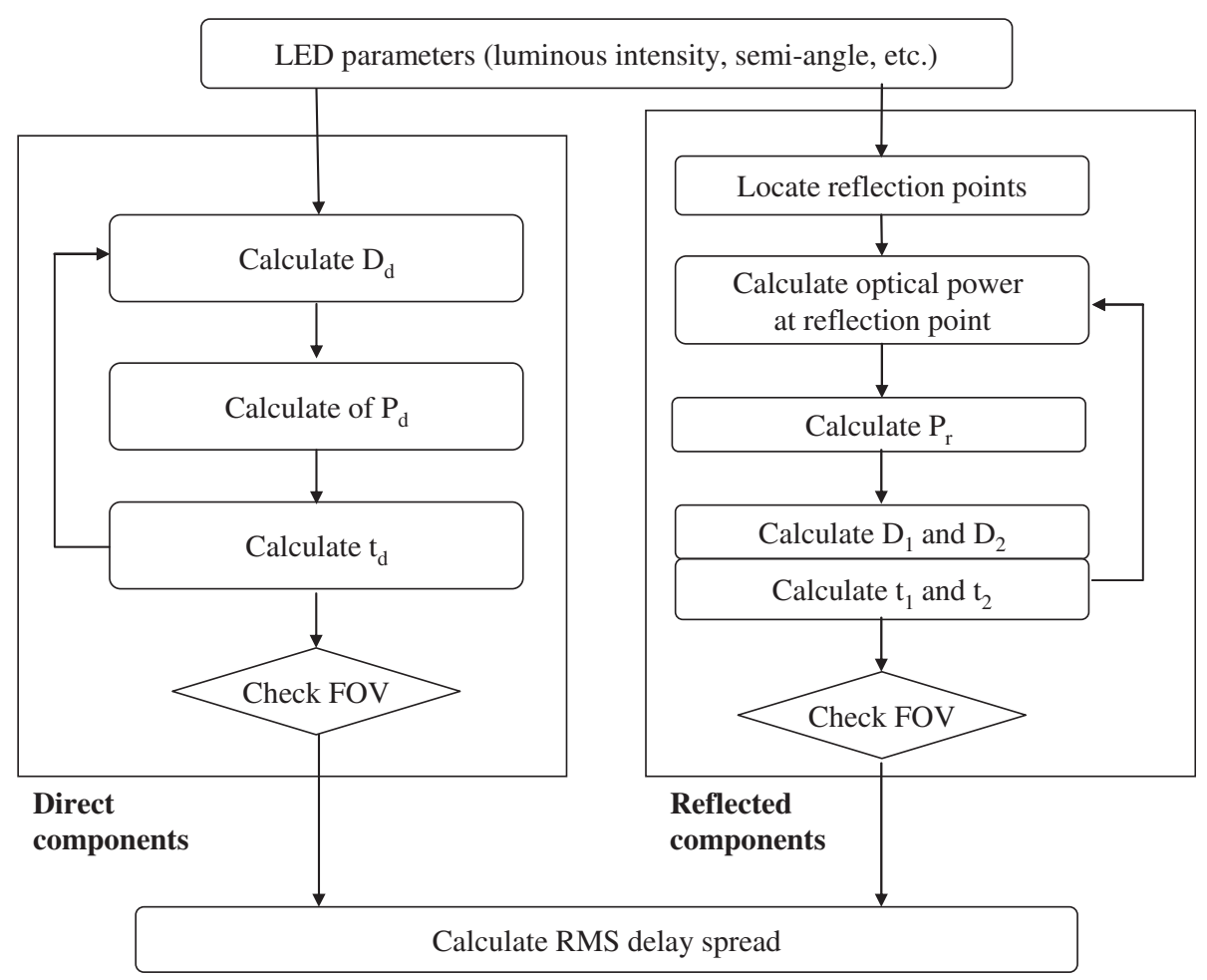

Figure 4 The flow chart used for calculating the RMSDS.

In order to evaluate the maximum transmission DR affected by the LED emission cross-section, the use of monochromatic light is assumed in this article. Therefore, from this monochromatic approximation, the distribution of the received optical power will be proportional to that of the illumination, and therefore, the signal-to-noise ratio (SNR) will show the same tendency as the horizontal illuminance distribution.

\section{Effect of emission cross-section on RMSDS}

We calculated the RMSDS distributions in order to estimate the effect of the emission cross-section on the DR. Figure 6 shows the RMSDS distributions for one transmitter; Figure 6(a) has a circular emission cross-section and Figure 6(b) has a non-circular quasi-elliptic emission cross-section. The maximum and minimum RMSDS values are summarized in Table 1.

For the single transmitter case, the minimum RMSDS for the non-circular quasi-elliptic $(0.1620 \mathrm{~ns})$ transmitter is larger than that found for the circular emission $(0.0040 \mathrm{~ns})$ transmitter, since the non-circular quasi-elliptic emission provides a wider illumination than the circular emission, thus generating more reflection components at the receiver.

Figure 7 shows the RMSDS distributions for four transmitters. The RMSDS values range from 0.3110 to $3.1886 \mathrm{~ns}$ for the circular emission, and from 0.3030 to $2.9868 \mathrm{~ns}$ for the non-circular quasi-elliptic emission. When using the four transmitters, the minimum RMSDS for the noncircular quasi-elliptic emission $(0.3030 \mathrm{~ns})$ is slightly lower than that found for the circular emission $(0.3110 \mathrm{~ns})$ since there are more chances for the light components to reach the receiver from the circular emission. With the increased number of transmitters, a decrease in the maximum RMSDS value and an increase in the minimum of RMSDS occur, since the strong light components are distributed more widely. The increased number of transmitters decreases the maximum values of RMSDS and increases the minimum values of RMSDS regardless of the emission cross-section shape. It should be noted that the difference between the maximum and minimum RMSDS values is reduced for the increased number of transmitters in both cases.

It is accepted that the maximum bit rate that can be transmitted through the channel without needing an equalizer will be limited to the DR determined by [29].

$$
R_{b} \leq 1 /\left(10 \times \tau_{R M S}\right) .
$$

At a certain point, the minimum RMSDS determines the maximum DR. However, on a whole receiving plane, the maximum RMSDS delay spread is also important since it corresponds to the minimum DR to be guaranteed over the 


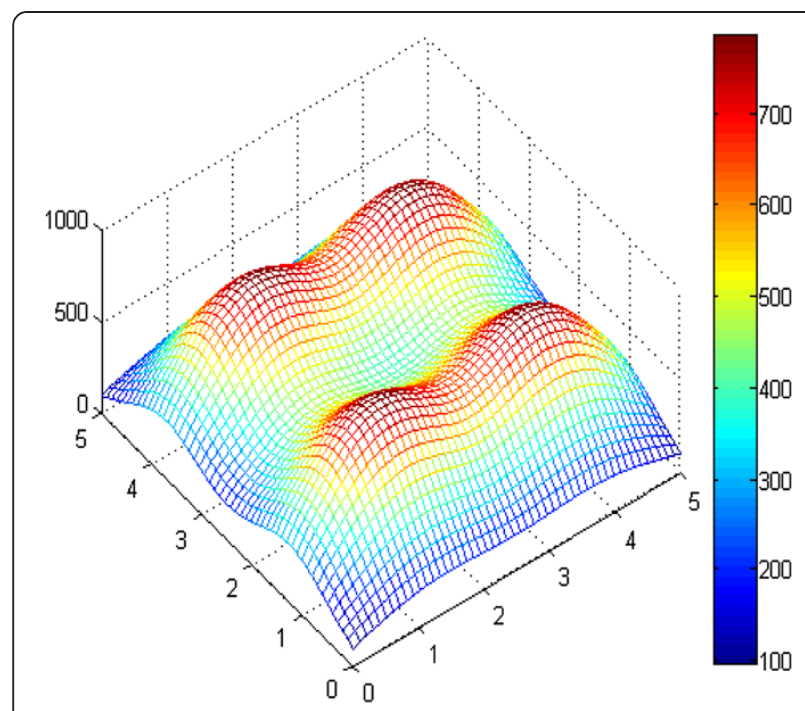

(a)

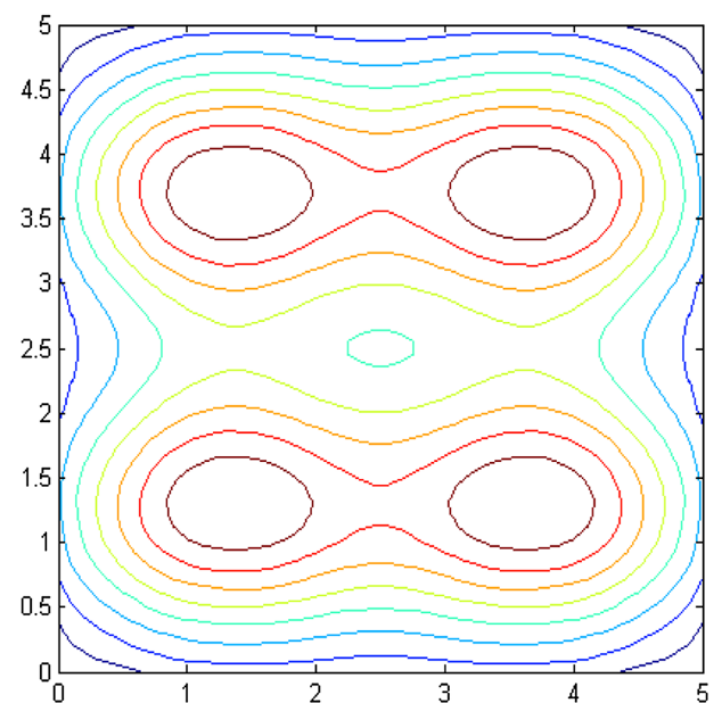

(b)

Figure 5 The distribution of the illuminance for 4-LED lamps with an elliptic emission (Max $=784.8996 \mathrm{Ix}, \mathrm{Min}=97.3568 \mathrm{Ix}$, Avg. $=\mathbf{4 8 1 . 6 4 2 6 ~ l x ) ~ : ~ ( a ) ~ t h e ~ m e s h ~ p l o t ; ~ ( b ) ~ t h e ~ c o n t o u r ~ p l o t . ~}$

whole plane. If a receiver is assumed to move around in a room, the minimum DR guaranteed over entire receiver plane will also be important in addition to the maximum DR at specific points. With one transmitter, it is found that the circular emission cross-section can operate over 15 $\mathrm{Mb} / \mathrm{s}$; the elliptic cross-section can operate over $24 \mathrm{Mb} / \mathrm{s}$. With four transmitters, it is found that the circular emission cross-section can operate over $30 \mathrm{Mb} / \mathrm{s}$; the elliptic cross-section can operate over $33 \mathrm{Mb} / \mathrm{s}$. These minimum DRs will be guaranteed for the entire receiving plane. The maximum DR at a certain point will be largest for the one transmitter with circular emission cross-section. The

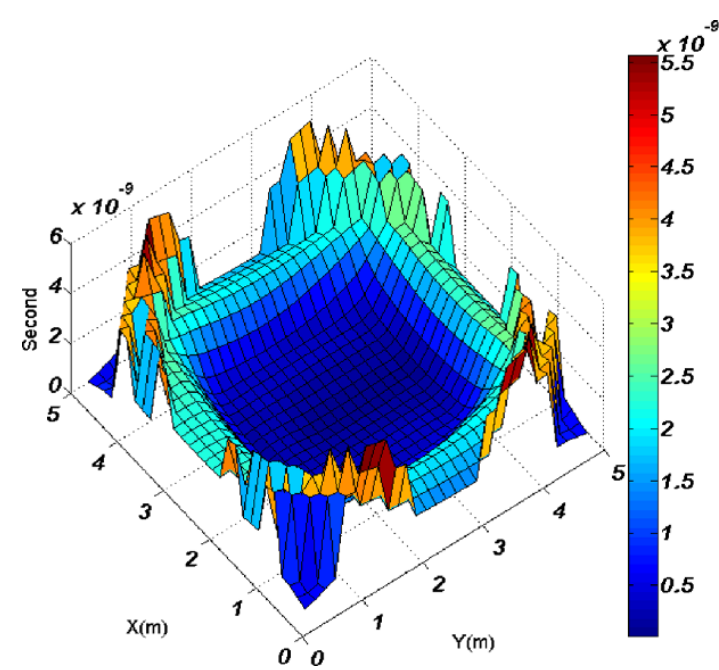

(a)

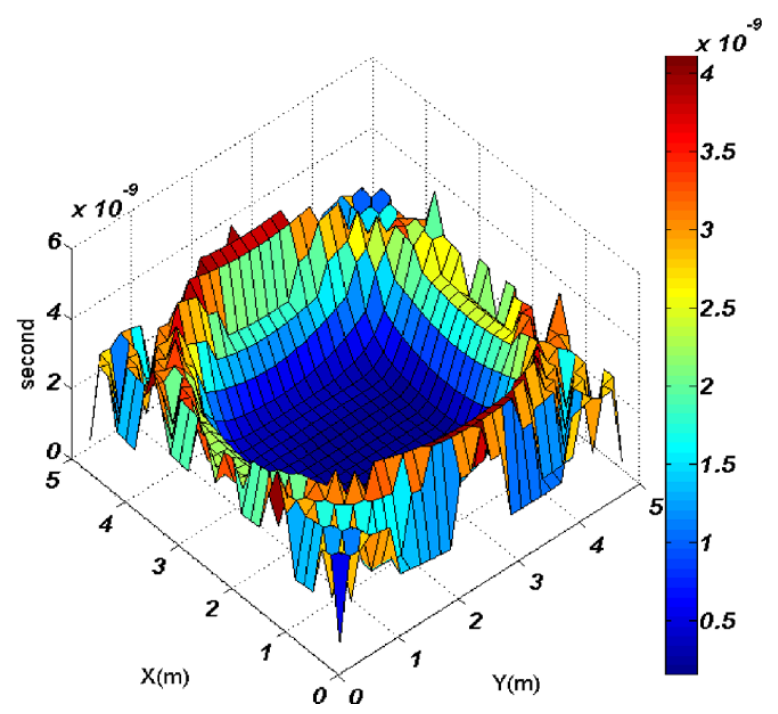

(b)

Figure 6 The RMSDS distribution for one transmitter: (a) circular emission and (b) non-circular emission with the position of the center LED at $(2.5,2.5)$.

difference due to emission cross-section becomes small in the four-transmitter case. If the number of transmitter increases, the gap between the maximum and minimum DRs for each case decreases since the rays spread out over the entire room surfaces, as predicted in Table 1.

Table 1 The Maximum and Minimum RMSDS Values

\begin{tabular}{cccccc}
\hline $\begin{array}{c}\text { RMSDS } \\
\text { (ns) }\end{array}$ & \multicolumn{2}{c}{ One transmitter } & & \multicolumn{2}{c}{ Four-transmitters } \\
\cline { 2 - 3 } \cline { 5 - 6 } & Circular & $\begin{array}{c}\text { Non-circular } \\
\text { (quasi-elliptic) }\end{array}$ & Circular & $\begin{array}{c}\text { Non-circular } \\
\text { (quasi-elliptic) }\end{array}$ \\
\hline Max. & 5.6409 & 4.1043 & & 3.1886 & 2.9868 \\
Min. & 0.0040 & 0.1620 & & 0.3110 & 0.3030 \\
\hline
\end{tabular}




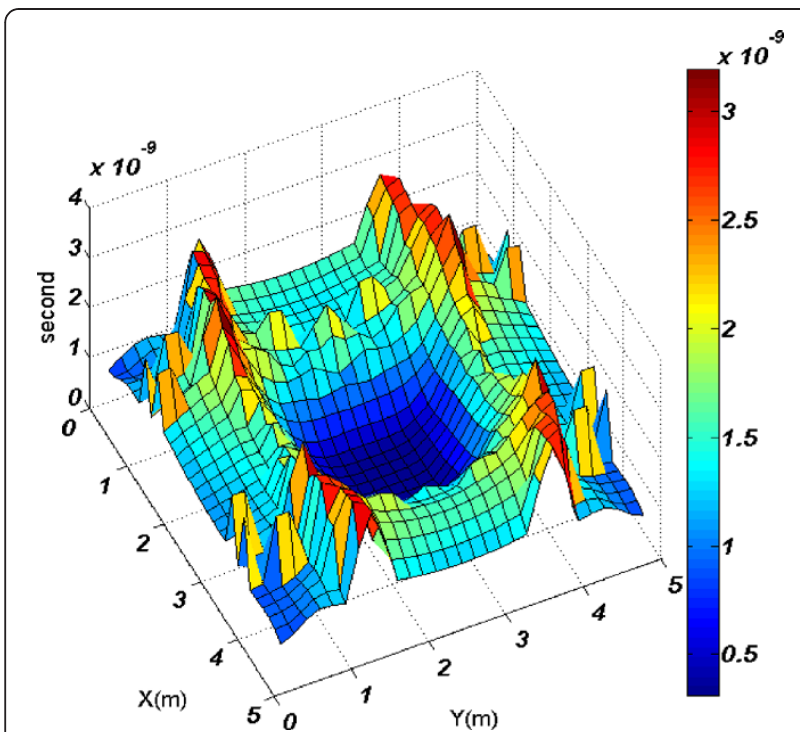

(a)

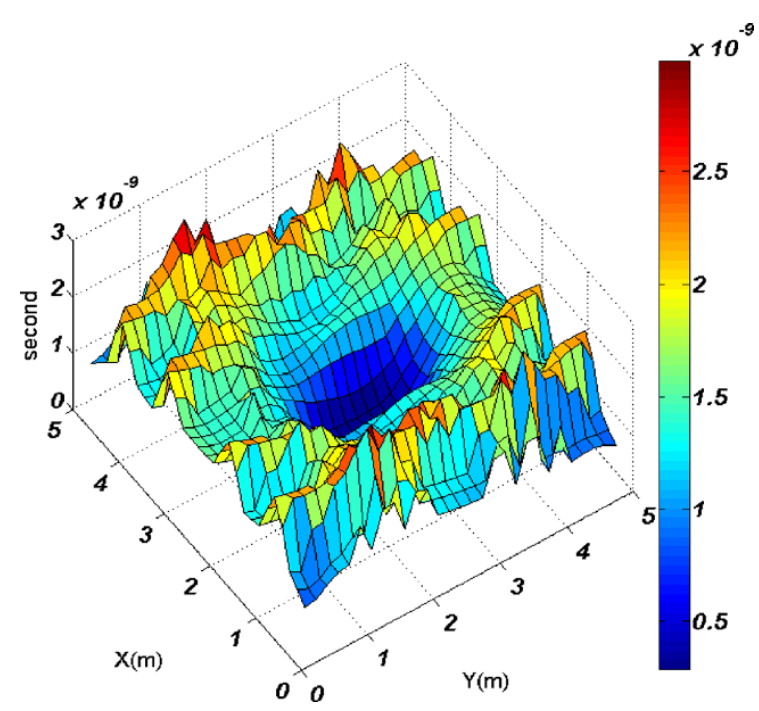

(b)

Figure 7 The RMSDS distribution for four transmitters: (a) circular emission and (b) non- circular emission with the positions of the transmitters at $(1.25,1.25),(1.25,3.75),(3.75$, $1.25)$, and $(3.75,3.75)$.

Usually, the illumination will be installed in a symmetrical manner. Therefore we used a symmetrical configuration in the room model. This creates redundancy. In order to avoid redundant RMSDS calculations using many sample points, we chose the simulation sample points as shown in Figure 8. The single LED transmitter was placed at the center $(2.5,2.5)$. The four LED lamp positions were $(1.25,1.25),(1.25,3.75)$, $(3.75,1.25)$, and $(3.75,3.75)$. The values from 1 to 9 in the $\mathrm{x}$-axies of Figure 9 denote the receiving

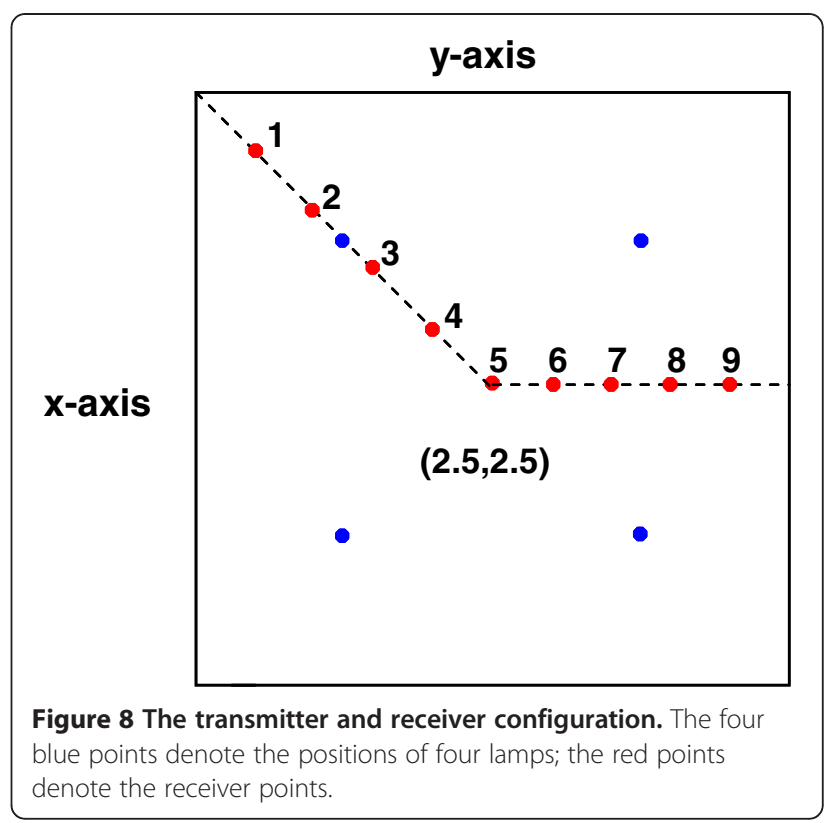

point positions. The nine sample points are chosen for showing results in various receiving points beyond symmetry since the room environment is symmetric. Therefore, the sample points were chosen following the diagonal lines from the left upper corner (position 1) to the center (position 5) and then to the rightmost position (position 9).

Figure 9 shows the changes in the RMSDS at different receiver positions. When the number of transmitters increases, the RMSDS increases and, consequently, the maximum DR becomes more limited. From an illumination viewpoint, a more uniform illumination can be achieved using an increased number of transmitters. A receiver position $(1 \rightarrow 5)$ along the diagonal line can effectively receive many reflected light components from the four walls and so obtains higher RMSDS values compared to the surrounding positions. As shown in Equation (7), the RMSDS is not zero if any delayed light component is present.

When we compare Figure 9(a) and 9(b), we notice that the RMSDS distribution is affected by the shape of the emission cross-section. Comparing Figure 9(a) to 9(b), the circular LED emission cross-section shows more drastic changes in the RMSDS for various positions than that found for the non-circular quasi-elliptic case, except in the center area. This is due to the strong concentration of optical power in the center area.

In the single transmitter case, the RMSDS values for the circular emission cross-section at the area near the walls (point 1) are larger than those found for the non-circular quasi-elliptic emission cross-section. In the four transmitter case, the RMSDS at position 1 is comparable to that at position 9 for the circular 


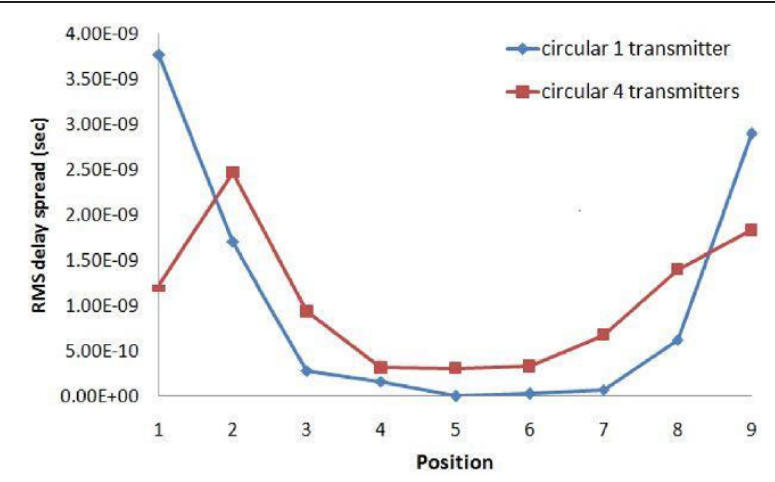

(a)

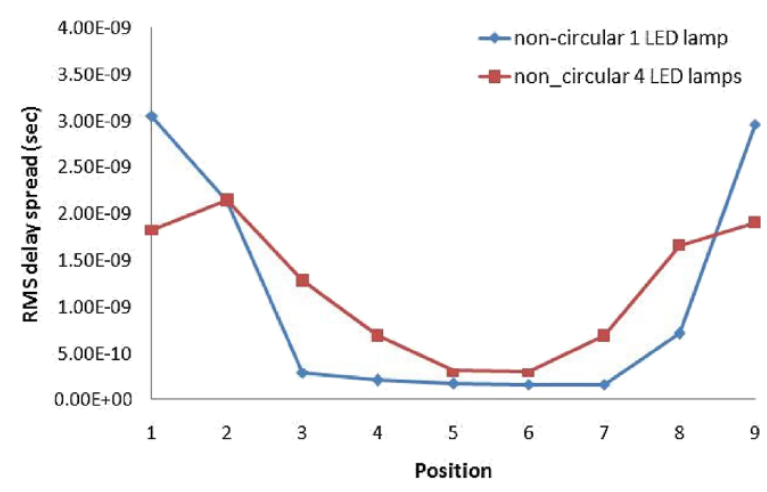

(b)

Figure 9 The RMSDS at several sample receiver positions: (a) circular and (b) non-circular emission cross-sections.

emission since the effect of the reflections is almost the same near the corner (position 1) and near the wall (position 9), regardless of the shape of the emission cross-section. This is because the light components are well distributed, even in the circular emission cross-section case. It should be noted that the distribution requirements regarding the circular emission cross-section transmitters is relaxed by using a large number of transmitters.

Regardless of the number and the emission crosssection type of the transmitters, the RMSDS increases near the corners and walls due to the long time delays for the light components, and thereby causing the maximum possible DR to be limited.

Up to this point, we have assumed that each LED transmitter is composed of a certain number of LEDs with an equivalent total optical power in order to determine the effects of the emission cross-section of an LED. Practically, however, many LEDs are used to form one lamp, as can be seen in [28]. The $5 \times 5 \mathrm{~m}$ LED transmitter array can be applied to design an LED illumination block by reducing the physical dimensions and the optical power of each LED.

\section{Discussions}

We have so far assumed that the LED transmitters emitted a monochromatic light. The optical power [Watt] of monochromatic light is related to the illuminance and is expressed as:

$$
P_{d i r}=\frac{I(0) \cdot \cos ^{m}(\phi)}{683 \cdot V(\lambda) \cdot D_{d}^{2} \cdot \cos (\psi)}
$$

where $\mathrm{V}(\lambda)$ is the eye sensitivity function [30], which is the conversion between the radiometric unit [Watt] and the photometric unit $[\mathrm{lm}]$ for a certain wavelength $\lambda . I(0)$ is the center luminous intensity of an LED light, $\phi$ is the angle of irradiance, $D_{d}$ is the distance between a center of LED group, $\psi$ is the angle of incidence to the receiver. At the wavelength of $555 \mathrm{~nm}$ (green), we have an eye sensitivity of $V(\lambda=555 \mathrm{~nm})=1$; at the wavelength of $720 \mathrm{~nm}$ (red), the eye sensitivity is $V(\lambda=720 \mathrm{~nm})=0.001$. The optical power of 1 [W] corresponds to the luminous flux of 683 lumen [lm] when the wavelength of the light is $555 \mathrm{~nm}$. The luminous flux of one lumen divided by the area of $1 \mathrm{~m}^{2}$ gives the illuminance of one lux [lx]. If the light is composed over a wide spectrum, the calculation must involve integration over the wavelengths occupied by the light.

Illumination LEDs provide multiple wavelengths in the visible range from 390 to $720 \mathrm{~nm}$. Therefore, the illuminance and received optical power calculations involve integration over the wavelengths occupied by the light and passed by the receiver optical filter:

$$
P_{d i r}=\frac{I(0) \cos ^{m}(\phi)}{683 D_{d}^{2} \cos (\psi) \int_{390}^{720} V(\lambda) P(\lambda) d \lambda}
$$

where $P(\lambda)$ is the power spectral density [30]. The other notations are the same as those denoted previously in this article.

Multiple wavelengths can also affect the light rays at different angles when reflected by rough wall surfaces. The optical lens used in concentrators or photodetectors can introduce additional aberrations. These effects will affects the RMSDS and, consequently limit the maximum DR. It is expected that the proposed model will be improved by adding simulation models for multiwavelength LEDs and wavelength-dependent reflection coefficients at wall surfaces. Since the RMSDS is a function of propagation time and optical power of each ray component, more precise results must be obtained considering multiple wavelength of the illumination LEDs, wavelength-dependent responsivity of receiver, and wavelength-dependent reflectance coefficients.

Including the reflection light, the expression for the total optical power would be modified from Equation (5) to a discrete form: 


$$
P_{\text {total }}=\sum^{L E D s} \sum^{\lambda}\left\{P_{\operatorname{dir}, \lambda}+\sum_{j}^{N} P_{r e f, \lambda, j}\right\} .
$$

To design reliable VLC systems installed in various indoor environments, it is necessary to investigate the communication performance according to the space dimensions, number of transmitters, optical parameters in receivers, etc. For example, the effect of the number of transmitters on RMSDS is as follows. With large number of transmitters, the maximum RMSDS is decreased and the minimum RMSDS is increased. For reliable communications in a room, the minimum available DR over the entire receiving plane is also important. The maximum RMSDS of the single transmitter case is significantly affected by room dimension. On the contrary, the effect of room dimension on the maximum RMSDS becomes weak in the case of four-transmitter. The large number of transmitters relaxes the conditions for reliable communication with the room dimension change.

The layout of room and illumination lights will be different in all cases, except apartment or other well-organized office rooms in a building. Therefore, simulation of various possible layouts will be very time consuming and makes it difficult to understand the effect of each parameter. The layout for a simulation can be very diverse. However, from the result of this article with two emission cross-section types (circular and quasi-elliptic) and two types of transmitter arrangements (one and four), it is noticed that the minimum DR guaranteed is higher for a case with non-circular emission cross-section and large number of transmitters.

\section{Conclusion}

We have presented a simple LED simulation model using a non-circular quasi-elliptic emission cross-section. By using the proposed model, we developed a simulation program based on MATLAB and Simulink. Using the simulation program, simulations have been performed for the distributions of illuminance, received optical power, and RMSDS for a non-circular quasi-elliptic emission crosssection, and compared to the results from a circular emission cross-section. The simulation results show that the emission cross-section affects the illumination and communication performance.

The LEDs with non-circular quasi-elliptic emission cross-sections show less fluctuation in illumination and optical power, however, the RMSDS was increased, subsequently limiting the maximum DR, compared to the LEDs with circular emissions. Therefore, we have shown that there must be a tradeoff between illumination and DR in the case of LED transmitters with non-circular quasi-elliptic emission cross-sections.
The LED transmitter with non-circular quasi-elliptic emission is preferred when it is used as a communication source since a minimum DR must be guaranteed over a whole service area. However, under a situation where a high DR transmission is required on a certain focused area, an LED transmitter with a circular emission is preferred. Thereby, we found that the emission cross-section of LEDs is one of the important design parameters to be considered for indoor VLC systems.

\section{Competing interests}

The authors declare that they have no competing interests.

\section{Acknowledgements}

This study was supported in part by the research funds from Chosun University, 2008.

\section{Author details}

${ }^{1}$ Department of Electronic Engineering, Chosun University, Gwangju 501-759, Korea. ${ }^{2}$ LED Communication Team, ETRI, Daejon 305-700, Korea. ${ }^{3}$ School of Computer Engineering, Chosun University, Gwangju 501-759, Korea.

Received: 17 April 2012 Accepted: 5 September 2012

Published: 12 September 2012

\section{References}

1. Bluetooth, http://www.bluetooth.org

2. IEEE 802, http://standards.ieee.org/getieee 802

3. S. Rajagopal, R.D. Roberts, S.-K. Lim, IEEE 802.15.7 visible light communication: modulation schemes and dimming support. IEEE Commun. Mag. 50, 72-82 (2012)

4. IrDA, http://www.irda.org

5. D. Porcino, W. Hirt, Ultra-wideband radio technology: potential and challenges ahead. IEEE Commun. Mag. 41, 66-74 (2003)

6. D.K. Borah, A.C. Boucouvalas, C.C. Davis, S. Hranilovic, K. Yiannopoulos, "A review of communication-oriented optical wireless systems". EURASIP J. Wirel. Commun. Netw. 91 (2012). doi:10.1186/1687-1499-2012-91

7. J. Vucic, C. Kottke, S. Nerreter, K.-D. Langer, J.W. Walewski, 513 Mbit/s visible light communications link based on DMT-modulation of a white LED. J. Lightwave Technol. 28, 3512-3518 (2010)

8. J. Rufo, J. Rabadan, F. Delgado, C. Quintana, R. Perez-Jimenez, Experimental evaluation of video transmission through LED illumination devices. IEEE Trans. Consumer Electron. 56, 1411-1416 (2010)

9. F. Delgado, I. Quintana, J. Rufo, J.A. Rabadan, C. Quintana, R. Perez-Jimenez, Design and implementation of an ethernet-VLC interface for broadcast transmissions. IEEE Commun. Lett. 14, 1089-1091 (2010)

10. A.M. Khalid, G. Cossu, R. Corsini, M. Presi, E. Ciaramella, Demonstrating a hybrid radio-over-fiber and visible light communication system. Electron. Lett. 47, 1136-1137 (2011)

11. M.F. Guerra-Medina, O. Gonzalez, B. Rojas-Guillama, J.A. Martin-Gonzalez, F. Delgado, J. Rabadan, Ethernet-OCDMA system for multi-user visible light communications. Electron. Lett. 48, 227-228 (2012)

12. D. O'Brien, R. Turnbull, H.L. Minh, G. Faulkner, O. Bouchet, P. Porcon, M.E. Tabach, E. Gueutier, M. Wolf, L. Grobe, J. Li, High-speed optical wireless demonstrators: conclusions and future directions. J. Lightwave Technol. 30, 2181-2187 (2012)

13. J.M. Kahn, J.R. Barry, Wireless infrared communications. Proc. IEEE 85, 265298 (1997)

14. J.B. Carruthers, P. Kannan, Iterative site-based modeling for wireless infrared channels. IEEE Trans. Antennas Propagat. 50, 759-765 (2002)

15. A. Sivabalan, J. John, Improved power distribution in diffuse indoor optical wireless systems employing multiple transmitter configurations. Opt. Quantum Electron. 38, 711-725 (2006)

16. Y. Tanaka, S. Haruyama, M. Nakagawa, Wireless optical transmissions with white colored LED for wireless home links, in Proceedings of the 11th Int. Symposium on Personal, Indoor and Mobile Radio Communications (PIMRC2000), ed. by, 2nd edn. (IEEE, London, UK, 2000). doi:10.1109/ PIMRC.2000.881634 
17. T. Komine, M. Nakagawa, Fundamental analysis for visible light communication system using LED light. IEEE Trans. Consum. Electron. 50 100-107 (2004)

18. T. Komine, S. Haruyama, M. Nakagawa, A study of shadowing on indoor visible-light wireless communication utilizing plural white LED lightings. Wirel. Personal Commun. 34, 211-225 (2005)

19. Y. Tanaka, T. Komine, S. Haruyama, M. Nakagawa, Indoor visible light data transmission system utilizing white LED lights. IEICE Trans. Commun. E86-B, 2440-2454 (2003)

20. C.G. Lee, C.S. Park, J.-H. Kim, D.-H. Kim, Experimental verification of optical wireless communication link using high-brightness illumination lightemitting diodes. Opt. Eng. 46, 125005 (2007)

21. D.W.K. Wong, G. Chen, Illumination design of a white light emitting diode wireless transmission system. Opt. Eng. 46, 085002 (2007)

22. H.L. Minh, D.C. O'Brien, G. Faulkner, L. Zeng, K. Lee, D. Jung, Y. Oh, Highspeed visible light communications using multiple-resonant equalization. IEEE Photon. Technol. Lett. 20, 1243-1245 (2008)

23. K.-D. Langer et al., Optical wireless communications for broadband access in home area networks, in Proceedings of the 10th Anniversary International Conference on Transparent Optical Networks (ICTON), ed. by, 4th edn. (IEEE, Athens, Greece, 2008), pp. 149-154. doi:10.1109/ICTON.2008.4598756

24. I. Moreno, M. Avendano-Alejo, R.I. Tzonchev, Designing light-emitting diode arrays for uniform near-field irradiance. Appl. Opt. 45, 2265-2272 (2006)

25. I. Moreno, C.-C. Sun, R. Ivanov, Far-field condition for light-emitting diode arrays. Appl. Opt. 48, 1190-1197 (2009)

26. I. Moreno, C.-C. Sun, Modeling the radiation pattern of LEDs. Opt. Express $16,1808-1819$ (2008)

27. C.-H. Tsuei, J.-W. Pen, W.-S. Sun, Simulating the illuminance and the efficiency of the LED and fluorescent lights used in indoor lighting design. Opt. Express 16, 18692-18701 (2008)

28. A.J.-W. Whang, Y.-Y. Chen, Y.-T. Teng, Designing uniform illumination systems by surface-tailored lens and configurations of LED arrays. J. Display Technol. 5, 94-102 (2009)

29. T.S. Rappaport, Wireless Communications: Principles and Practice, 2nd edn. (Prentice-Hall, Upper Saddle River, NJ, USA, 2002)

30. E.F. Schubert, Light-emitting Diodes, 2nd edn. (Cambridge University Press, Cambridge, UK, 2006)

doi:10.1186/1687-1499-2012-286

Cite this article as: Nguyen et al:: Effect of LED emission cross-section in indoor visible light communication systems. EURASIP Journal on Wireless Communications and Networking 2012 2012:286.

\section{Submit your manuscript to a SpringerOpen ${ }^{\circ}$ journal and benefit from:}

- Convenient online submission

- Rigorous peer review

- Immediate publication on acceptance

- Open access: articles freely available online

- High visibility within the field

- Retaining the copyright to your article

Submit your next manuscript at $\gg$ springeropen.com 